\title{
2012 Steele Prizes
}

The 2012 AMS Leroy P. Steele Prizes were presented at the 118th Annual Meeting of the AMS in Boston in January 2012. The Steele Prizes were awarded to MICHAEL ASCHBACHER, RICHARD LyONS, STEVE SMITH, and RonAld SOLOMON for Mathematical Exposition; to WILLIAM THURSTON for a Seminal Contribution to Research; and to IVO M. BABUŠKA for Lifetime Achievement.

\section{Mathematical Exposition: Michael Aschbacher, Richard Lyons, Steve Smith, and Ronald Solomon}

\section{Citation}

The 2012 Leroy P. Steele Prize for Mathematical Exposition is awarded to Michael Aschbacher, Richard Lyons, Steve Smith, and Ronald Solomon for their work, The Classification of Finite Simple Groups: Groups of Characteristic 2 Type, Mathematical Surveys and Monographs, 172, American Mathematical Society, Providence, RI, 2011. In this paper, the authors, who have done foundational work in the classification of finite simple groups, offer to the general mathematical public an articulate and readable exposition of the classification of characteristic 2 type groups.

\section{Biographical Sketches}

Michael Aschbacher was born in Little Rock, Arkansas, in 1944. He received his undergraduate degree from Caltech in 1966 and his Ph.D. from the University of Wisconsin in 1969 under the direction of Richard Bruck. He was a postdoctoral fellow at the University of Illinois in 1969-70, and since then he has been at Caltech, where he is the Shaler Arthur Hanisch Professor of Mathematics. He received the Cole Prize in Algebra from the AMS in 1980 and the Rolf Schock Prize from the Royal Swedish Academy of Sciences in 2011. He was an invited speaker at the International Congress of Mathematicians in 1978 and a vice president of the AMS from 1996

DOI: http://dx.doi.org/10.1090/noti826 to 1998. He is a member of the National Academy of Sciences and the American Academy of Arts and Sciences. Aschbacher's research focuses on the finite simple groups.

Richard Lyons earned his Ph.D. under the supervision of John G. Thompson at the University of Chicago, with a brief stop at the University of Cambridge. At Chicago he had further tutelage from Jon Alperin, Richard Brauer, George Glauberman, Marty Isaacs, and Leonard Scott. He had graduated from Harvard College and had been inspired by the high school teaching of Dr. Beryl E. Hunte. After a J. Willard Gibbs Instructorship at Yale, he joined the faculty of Rutgers, where he began a long-term collaboration with the late Danny Gorenstein and where he now serves in his fortieth year.

Stephen D. Smith is Professor Emeritus of Mathematics at the University of Illinois at Chicago. He received his S.B. from M.I.T. in 1970 and his D.Phil. in 1973 under the supervision of Graham Higman at Oxford (where he was a Rhodes Scholar). He was a Bateman Research Instructor at Caltech from 1973 to 1975 and then moved to UIC as assistant professor and later associate professor and professor. He has published mainly in finite group theory, with further interests in combinatorics, algebraic topology, and computer science. In addition to the book cited for this Steele Prize, which was written jointly with Aschbacher, Lyons, and Solomon, he has also published The Classification of Quasithin Groups with Michael Aschbacher, Classifying Spaces of Sporadic Groups with Dave Benson, and most recently Subgroup Complexes. Smith married Judith L. Baxter in 1980 and has two adult stepchildren.

Ronald Solomon got his love of words from his mother and his love of math from his high school teacher, Blossom Backal. He graduated from Queens College (CUNY) in 1968 and earned his Ph.D. at Yale University in 1971 under the supervision of Walter Feit. After a Dickson Instructorship at the University of Chicago and a year at Rutgers University, he joined the faculty of the Ohio State 


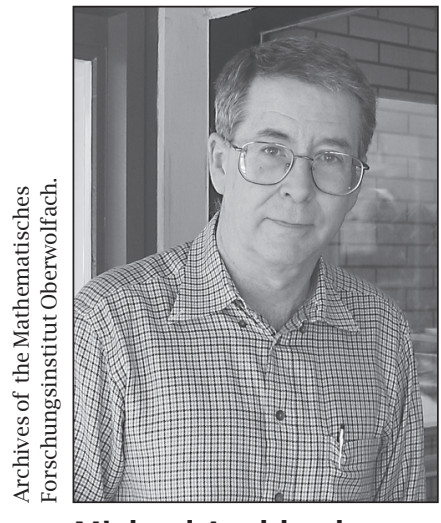

Michael Aschbacher

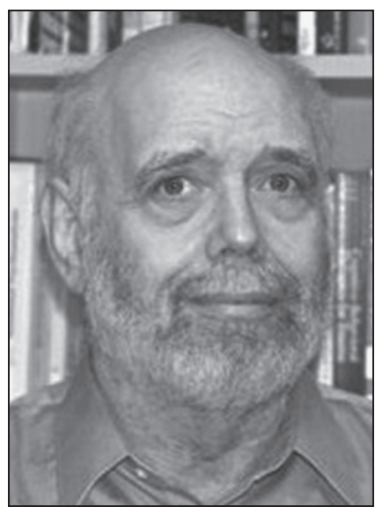

Richard Lyons

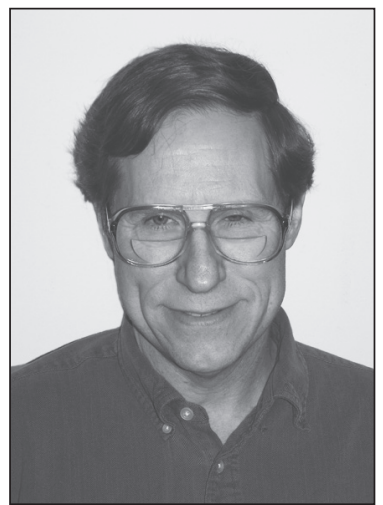

Steve Smith

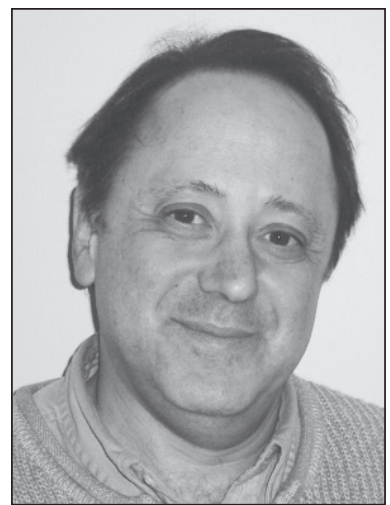

Ronald Solomon

University in 1975. Since 1982 he has been a member of a team, with Danny Gorenstein and Richard Lyons, that wrote a series of volumes (Mathematical Surveys and Monographs, 40.1-40.6, American Mathematical Society, Providence, RI, 1994, 1996, 1998, 1999, 2002, 2005) which presents a substantial portion of the proof of the classification of the finite simple groups. Ron earned an Ohio State Distinguished Teaching Award in 1997 and the Levi L. Conant Prize of the AMS in 2006. He is grateful and proud to be the husband of Rose and the father of Ari and Michael.

\section{Joint Response from Michael Aschbacher, Richard Lyons, Steve Smith, and Ronald Solomon}

We are deeply grateful to the Society for honoring us with this Steele Prize for Mathematical Exposition. For decades Danny Gorenstein was the voice of the Classification Project, providing the community with a vivid narrative of our travails and accomplishments. Unfortunately, he departed this life before the task was completed and the tale fully told. Our book serves in part as a sequel to his 1983 volume, providing a detailed reader's guide to the major papers composing the second ("even") half of the Classification proof, but we have prefaced it with an outline and synopsis of the entire proof, updating Danny's references and giving our personal view of the entire enterprise. In writing a book it always helps to have a great story to tell, and few mathematical projects have played out on such an epic scale and reached such a gratifying culmination as the Classification of the Finite Simple Groups. We appreciate that, in awarding us this prize, the Society acknowledges the importance of this work.

\section{Seminal Contribution to Research: William Thurston}

\section{Citation}

The Leroy P. Steele Prize for Seminal Contribution to Research is awarded to William Thurston for his

contributions to low dimensional topology, and in particular for a series of highly original papers, starting with "Hyperbolic structures on 3-manifolds. I. Deformation of acylindrical manifolds" (Ann. of Math. (2) 124 (1986), no. 2, 203-246), that revolutionized 3-manifold theory. These papers transformed the field from a subfield of combinatorial topology to a web of connections between topology, complex analysis, dynamical systems, and hyperbolic geometry. In addition, Thurston not only gave a complete conjectural picture of all compact 3-manifolds, but in these papers he proved his conjecture for a large class of examples, namely Haken manifolds, which include all compact 3-manifolds with nonempty boundary.

\section{Biographical Sketch}

William P. Thurston was born October 30, 1946, in Washington, DC, and he received his Ph.D.

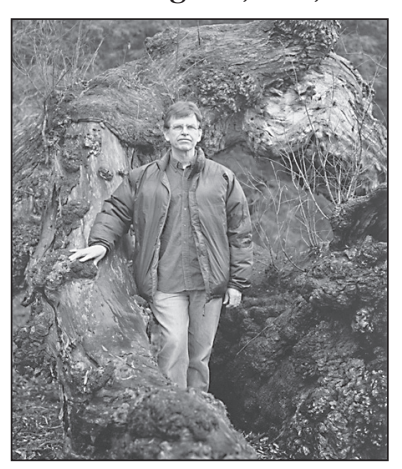
in mathematics from the University of California at Berkeley in 1972. He taught at the IAS (1972-73) and at MIT (1973-74) before joining the faculty of Princeton University in 1974. Professor Thurston returned to UCBerkeley, this time as a faculty member, in 1991 and became director of MSRI in 1993. He William Thurston

then taught at UC-Davis from 1996 to 2003 and accepted a position at Cornell University in 2003, where he holds joint appointments in the Department of Mathematics and the Faculty of Computing and Information Science.

Professor Thurston held an Alfred P. Sloan Foundation Fellowship in 1974-1975; in 1976 he was awarded the AMS Oswald Veblen Geometry Prize for his work on foliations. In 1979 he became the second mathematician ever to receive the Alan T. Waterman Award, and in 1982 Professor 
Thurston was awarded the Fields Medal. He is a member of the American Academy of Arts and Sciences and the National Academy of Sciences.

\section{Response}

I am deeply honored by this recognition from the American Mathematical Society. I have loved mathematics all my life. I felt very lucky when I discovered the mathematical community-local, national, and international-starting in graduate school. So the Steele Prize, with its long and distinguished history of honoring mathematicians whom I greatly admire, means a lot to me.

The work cited by the Steele Prize focuses on what I called the "geometrization conjecture". When I gradually realized the geometric beauty of 3-manifolds, it was as if a giant whirlwind, far bigger and far stronger than I, had swept me up and taken over my mathematical life. I couldn't escape (admittedly, I didn't even want to escape). At first I glimpsed only parts of the big picture, but little by little it came into focus and the mist blew away. I worked very hard and was able to prove the geometrization conjecture in many important cases, including, in some sense, "almost all" cases. I became completely convinced that the geometrization conjecture is true, but my approaches were extremely difficult, if not impossible, to push through.

I was ecstatic to be able to prove the geometrization conjecture in certain sweeping families of cases, but I was frustrated that my various methods seemed very difficult, if not impossible, to extend to all cases. I became completely convinced that the geometrization theorem was true, but it was frustrating not to have a complete proof. I was very pleased when Grigori Perelman, using very natural methods pioneered by Richard Hamilton (but foreign to my technical expertise) proved the geometrization conjecture in full generality.

I have been very lucky to have a long stream of wonderful students. They and others have built up a thriving mathematical community well versed in geometric structures on 3-manifolds, as well as other related structures on 3-manifolds, such as taut foliations, tight contact structures, etc. There are still many mysteries to solve in this area. I used to feel that there was certain knowledge and certain ways of thinking that were unique to me. It is very satisfying to have arrived at a stage where this is no longer true-lots of people have picked up on my ways of thought, and many people have proven theorems that I once tried and failed to prove.

\section{Lifetime Achievement: Ivo M. Babuška}

\section{Citation}

The 2012 Steele Prize for Lifetime Achievement is awarded to Ivo M. Babuška for his many pioneering advances in the numerical solution of partial differential equations over the last half century.

In his work on finite element methods, Babuška has developed and applied mathematics in profound ways to develop, analyze, and validate algorithms which are crucial for computational science and engineering. In so doing, he has helped to define that field and has had a great impact on the modern world.

A constant characteristic of Babuška's work is the combination of deep and imaginative math-

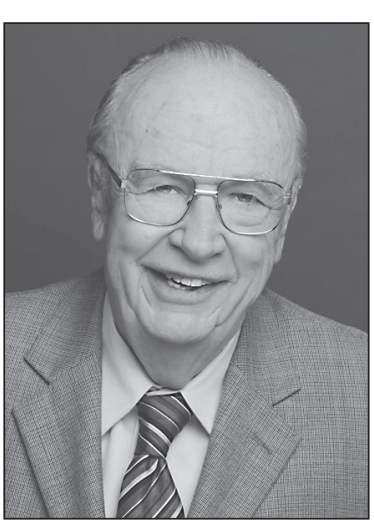

Ivo Babuška ematical analysis with a constant concern for the practical implications of his work for engineering applications. In seminal work of the 1960s and 1970s, he established the mathematical foundations of the finite element method culminating in a monumental and highly influential treatise coauthored with Aziz in 1972. In this early work he established the essential role of stability of Galerkin methods and formulated the discrete inf-sup condition, later to be named the Babuška-Brezzi condition, and developed the approximation theory of finite element spaces. He also introduced many techniques of lasting importance, such as the imposition of Dirichlet boundary conditions through Lagrange multipliers and through penalties, analysis through mesh-dependent norms, the first studies of a posteriori error estimation, and the Babuška-Rheinboldt theory of adaptivity. The Babuška paradox for elastic plates, which shows strikingly that the deformation of a circular elastic plate is not well approximated by the deformation of even a nearly equal polygonal plate, has inspired important developments in mechanics, partial differential equations, and numerical methods.

Babuška is an exceptionally productive author, collaborator, and mentor. He has published over 350 refereed journal articles and 26 books, has had nearly 150 coauthors, and has advised 40 Ph.D. students. An astounding feature of Babuška's work is how many themes he initiated [that] grew into large and active research fields. In the mid-1970s Babuška was among the pioneers of homogenization, which aims to capture the large-scale effects of fine-scale features of materials without resolving them. Later he developed generalized finite element methods with J. E. Osborn which sought to capture the influence of subgrid scale features in computational methods and anticipated a large and currently active branch of research in multiscale numerical methods. In the late 1980s 
Babuška and collaborators developed the $p$-version of the finite element method, and later the $h p$ method, and developed an elaborate theory for understanding its convergence in the presence of singularities. His important work in dimensional reduction and hierarchical models also dates from this time. In the 1990s, he developed the partition-of-unity finite element method, which led to another large and active area of development on meshless methods. In this century he has led the way to the computation of partial differential equations with uncertain data and the booming field of uncertainty quantification.

Ivo M. Babuška is among the foremost numerical analysts of all time and a unique leader in applied mathematics. His many contributions have had a lasting impact on mathematics, engineering, science, and industry. The Steele Prize honors him for all of these achievements.

\section{Biographical Sketch}

Ivo Babuška was born 1926 in Prague, Czechoslovakia. He received his civil engineering degree (Ing.) and his Ph.D. degree from the Czech Technical University in Prague. After that he studied mathematics and received a Ph.D. (then called a Candidate of Science, C.Sc., degree as in the USSR) and then the doctorate degree (Doctor of Science, D.Sc.) in mathematics from the Czechoslovak Academy of Sciences. He worked in the Mathematical Institute of the Academy and received the Czechoslovak State Award for his scientific work in 1968.

In 1968 he came to the University of Maryland at College Park as a visiting scientist, where he then became a professor in the mathematics department. He retired from Maryland as Distinguished University Professor in 1995.

Since 1995 he has been a senior scientist of the Institute for Computational Engineering and Sciences and professor of aerospace engineering and engineering mechanics, holding the Robert Trull Chair in Engineering at the University of Texas at Austin. Now half retired, he is still working at the university.

Ivo Babuška has received various honors recognizing his contributions. He has been awarded five honorary doctorate degrees, was elected to the U.S. National Academy of Engineering, the European Academy of Sciences, the Engineering Academy of the Czech Republic, and the Learned Society of Czech Republic. He is a fellow of SIAM and ICAM. Asteroid 36060 was named Babuška. He also received various recognitions, including the Birkhoff Prize of SIAM and the AMS, the ICAM Congress Medal, and the Bolzano Medal.

\section{Response from Ivo Babuška}

I am deeply honored to receive the Steele Prize for Lifetime Achievement from the AMS because my work encompasses both mathematics and engineering applications and computations. I am very fortunate that many of my mathematical results are used widely in engineering and practical computations. It is very satisfactory and important to me that my mathematical results are appreciated by both the mathematical and engineering communities and [that] they are also used in practice. This was influenced by my combined education in engineering and mathematics and by my mentors, Professor Faltus in engineering, Professor E. Cech, the well-known topologist, and Professor F. Vycichlo, to whom I am very grateful. On this occasion, I would like to thank all my scientific collaborators and friends in the mathematics and engineering communities. I cannot list them all, so I will mention here only a very few: J. Osborn, J. Whiteman, J. T. Oden, and B. Szabo, and my students with whom I have enjoyed not only doing mathematics but also hiking, skiing, and various excursions and adventures.

Finally, I would like to thank very much the selection committee for this great honor.

\section{About the Prize}

The Steele Prizes were established in 1970 in honor of George David Birkhoff, William Fogg Osgood, and William Caspar Graustein. Osgood was president of the AMS during 1905-06, and Birkhoff served in that capacity during 1925-26. The prizes are endowed under the terms of a bequest from Leroy P. Steele. Up to three prizes are awarded each year in the following categories: (1) Lifetime Achievement: for the cumulative influence of the total mathematical work of the recipient, high level of research over a period of time, particular influence on the development of a field, and influence on mathematics through Ph.D. students; (2) Mathematical Exposition: for a book or substantial survey or expository research paper; (3) Seminal Contribution to Research: for a paper, whether recent or not, that has proved to be of fundamental or lasting importance in its field or a model of important research. Each Steele Prize carries a cash award of US $\$ 5,000$.

Beginning with the 1994 prize, there has been a five-year cycle of fields for the Seminal Contribution to Research Award. For the 2012 prize, the field was geometry/topology. The Steele Prizes are awarded by the AMS Council acting on the recommendation of a selection committee. For the 2012 prizes, the members of the selection committee were Peter S. Constantin, Yakov Eliashberg, John E. Fornaess, Irene M. Gamba, Barbara L. Keyfitz, Joel A. Smoller, Terence C. Tao, Akshay Venkatesh, and Lai-Sang Young. The list of previous recipients of the Steele Prize may be found on the AMS website at http://www.ams.org/prizes-awards.

- Elaine Kehoe 